\title{
An Approach to Identification of Dynamic Plants Described by Irrational Transfer Functions
}

Anisimov, D., Zhelbakov, I. and Houlden, N.

This is a paper presented at the 7th IEEE Int. Conference on Internet Technologies and Applications ITA-17, Wrexham, UK, 12-15 September 2017.

Copyright of the author(s). Reproduced here with their permission and the permission of the conference organisers.

\section{Recommended citation:}

Anisimov, D., Zhelbakov, I. and Houlden, N. (2017) 'An Approach to Identification of Dynamic Plants Described by Irrational Transfer Functions'. In: Proc. 7th IEEE Int. Conference on Internet Technologies and Applications ITA-17, Wrexham, UK, 12-15 September 2017, pp. 177-180. doi: 10.1109/ITECHA.2017.8101933 


\section{An Approach to Identification of Dynamic Plants Described by Irrational Transfer Functions}

\author{
Dmitry Anisimov, Igor Zhelbakov \\ Institute of Automatics and Computer Engineering \\ National Research University "MPEI" \\ Moscow, Russia
}

\begin{abstract}
In this paper one of approaches to parametric identification of plants with distributed parameters is considered. The approach is based on the exponential modulation method (EMM). This method, originally developed for objects with lumped parameters and described by rational transfer functions, is extended for identification of plants described by irrational transfer functions. As an example, the process of radiation heating of the solid is considered for various boundary conditions. Identification is carried out according to the noisy transition processes. The comparison of the transients at the outputs of the object and model is given.
\end{abstract}

Keywords-identification, plant with distributed parameters, irrational transfer function, heat conduction equation, boundary conditions

\section{INTRODUCTION}

Irrational transfer functions have been widely used for the modelling of some physical and real-world phenomena [1]. They are obtained from linear distributed-parameter systems. Heat transfer processes [2], The diffusion approximation for the Boltzmann (transport) equation [3], modelling of hydropower plants [4, 5], lossy transmission lines [6], the analysis of the global warming [7] are some of dynamical systems that are described by irrational transfer functions.

It is clear that there are basic differences between rational and irrational transfer functions. For example, irrational transfer functions cannot be rewritten in a finite-dimensional state-space form, or they generally have infinitely many poles or zeros [8]. According to these differences, parametric identification of dynamic plants described by irrational transfer functions seems more complicated problem than identification of plants described by fractional rational 1 transfer functions. An approach for solving this problem is considered in the paper. This approach is based on the socalled exponential modulation method (EMM) [9]. It is similar to the well-known modulating function method (MFM) [10, 11]. They both use special formed modulating functions and calculation of areas under curves. The last feature provides high noise immunity of parameters estimation. The main difference between these two methods is following. MFM is based on integration of ordinary differential equations, while EMM uses Laplace transform of input and output signals. The description of the object in the form of the transfer function makes possible to estimate parameters of distributedparameter systems.

\author{
Nigel Houlden \\ School of Applied Science, Computing and Engineering \\ Glyndwr University \\ Plas Coch, Mold Road, Wrexham, LL11 2AW, UK
}

\section{ExPONENTIAL Modulation Method}

Since the method of exponential modulation is not covered widely in the literature, we will give its brief description. EMM was originally developed for linear systems described by fractional rational transfer function

$$
G(s)=\frac{Y(s)}{X(s)} \frac{b_{m} s^{m}+b_{m-1} s^{m-1}+\cdots+b_{0}}{a_{n} s^{n}+a_{n-1} s^{n-1}+\cdots+a_{1}+1}, m \leq n
$$

where $X(s)$ and $Y(s)$ are Laplace representation of input and output plant signals, $b_{0}, \ldots b_{m}, a_{1}, \ldots a_{n}-$ the unknown parameters that must be defined. Let at time $t=0$ the signal $x(t)$ begins to operate at the input of the plant. The input and output signals $x(t)$ and $y(t)$ are measured in time interval from 0 to $T_{H}$. Let us form such the function $\psi(t)=\exp (-t / \theta)$ that $\psi\left(T_{H}\right)<<1$, multiple it on the signals $x(t)$ and $y(t)$, and calculate the areas $S_{x}$ and $S_{y}$ under the curves formed (Fig. 1). Then

$$
\begin{aligned}
S_{x} & =\int_{0}^{T_{H}} x(t) \psi(t) d t \approx \int_{0}^{\infty} x(t) \psi(t) d t= \\
& =\int_{0}^{\infty} x(t) \exp (-t / \theta) d t=X\left(\theta^{-1}\right) \\
S_{y} & =\int_{0}^{T_{H}} y(t) \psi(t) d t \approx \int_{0}^{\infty} y(t) \psi(t) d t= \\
& =\int_{0}^{\infty} y(t) \exp (-t / \theta) d t=Y\left(\theta^{-1}\right)
\end{aligned}
$$

In this formula $X\left(\theta^{-1}\right)$ and $Y\left(\theta^{-1}\right)$ are Laplace representation of input and output signals that would be obtained by substituting the real value $\theta^{-1}$ instead Laplace operator $s$. We will call variable $\theta$ "the time constant of the modulating function (TCMF)".

As follows from (1) and (2),

$$
G\left(\theta^{-1}\right)=\frac{b_{m} \theta^{-m}+b_{m-1} \theta^{-m+1}+\cdots b_{1} \theta^{-1}+b_{0}}{a_{n} \theta^{-n}+a_{n-1} \theta^{-n+1}+\cdots a_{1} \theta^{-1}+1}=\frac{S_{y}}{S_{x}}
$$

This expression establishes a relationship between TCMF, calculated areas $S_{x}, S_{y}$ and plant parameters. Obviously, in order to determine all the parameters of the plant, we need to form $q=m+n+1$ modulating functions. Then the parameters of the plant can be determined from the solution of a system of linear equations:

$$
\mathbf{C}=\boldsymbol{\Gamma}^{-1} \Theta
$$


where

$$
\begin{aligned}
\mathbf{C} & =\left[\begin{array}{lllllc}
a_{n} & \cdots & a_{1} & b_{m} & \cdots & b_{0}
\end{array}\right]^{T}, \boldsymbol{\Theta}=\left[\begin{array}{ccccc}
-\theta_{1}^{n} & \cdots & -\theta_{q}^{n}
\end{array}\right]^{T} \\
\boldsymbol{\Gamma} & =\left[\begin{array}{ccccccc}
1 & \theta_{1} & \cdots & \theta_{1}^{n-1} & -d_{1} \theta_{1}^{n-m} & \cdots & -d_{1} \theta_{1}^{n} \\
\cdots & \cdots & \cdots & \cdots & \cdots & \cdots & \cdots \\
\cdots & \cdots & \cdots & \cdots & \cdots & \cdots & \cdots \\
1 & \theta_{q} & \cdots & \theta_{q}^{n-1} & -d_{q} \theta_{q}^{n-m} & \cdots & -d_{q} \theta_{q}^{n}
\end{array}\right], d_{i}=\frac{S_{x i}}{S_{y i}}
\end{aligned}
$$

If identification is carried out in ideal conditions (the observation time is not limited, sampling increment is infinitesimal, noise is absent), the errors of the parameters estimation is zero irrespective of the input signal and selecting TCMF. In practice neither of these conditions is met. The main source of error is the noise acting on the plant. Under the current disturbance, the error depends on the input signal and the time constants of the modulating functions. In fact, if the signal level at the input of the object is small, then the output level will be small too. Against this background, the influence of the noise can be significant. As shown in [12], EMM provides a better estimation of the parameters, if the input signal is the step signal of the maximum permissible level. TCMF should be set from the following considerations. On the one hand, they cannot be selected too small because the values of the calculated areas in this case would be uninformative due to the large specific weight of the random noise. On the other hand, they should not be too large, because the modulating the functions will not have time to damp by the end of the observation process. This can lead to the fact that the approximate equality in (2) would not be correct and the regular error in calculating of the areas will appear.

\section{IDENTIFICATION OF THE PLANT WITH DISTRIBUTED PARAMETERS}

The specificity of the exponential modulation method makes possible parametric identification of the plants described by irrational transfer functions. As noted above, such a mathematical description is typical for plants with distributed parameters. It can be obtained by solving the partial differential equations for fixed spatial coordinates.

In this paper we will consider the plant described by heat conduction equation. Let us consider the radiation heating of solid of unlimited thickness. Assume firstly that there are no heat elimination from the surface.

The thermal regime of the heating of the material surface can be approximated by one-dimensional Fourier equation for semi-bounded solid:

$$
\partial^{2} v(r, t) / \partial r^{2}=a(\partial v(r, t) / \partial t)
$$

where $v(r, t)$ is the solid temperature at time $t$ at position $r$, $a=\rho C_{p} / K_{0}, K_{0}$ is the thermal conductivity, $\rho$ is the mass density and $C_{p}$ is the specific heat capacity. As an example, consider a temperature change at a depth $l=0.1 \mathrm{~m}$ by heating of semi-bounded copper ingot. Then $K_{0}=401 \mathrm{~W} /(\mathrm{m} \cdot \mathrm{K})$, $\rho=8920 \mathrm{~kg} / \mathrm{m}^{3}, C_{p}=385 \mathrm{~J} /(\mathrm{kg} \cdot \mathrm{K}), a=8564 \mathrm{~s} / \mathrm{m}^{2}$. The form of irrational transfer function corresponding to equation (5) depends significantly on the boundary conditions, taking into

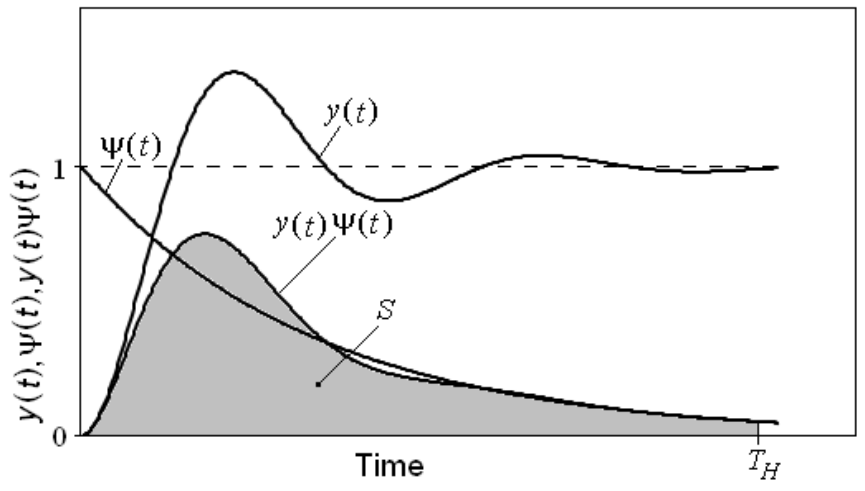

Fig. 1. The process at the output of the plant modulating a function of and their product.

account the input and the measuring point of the output signal. Let us consider the value $v$ as a sine wave varying with frequency $\omega$. Its phasor is

$$
V(i \omega t)=V_{m} \mathrm{e}^{i \omega t}
$$

Then equation (5) can be transformed in the following way:

$$
d^{2} V_{m} / d r^{2}-i \omega a V_{m}=0
$$

This is a homogeneous differential equation having the roots of the characteristic equation

$$
\gamma_{1,2}= \pm \sqrt{i \omega a}
$$

The solution of equation (7) has the form

$$
V_{m}=A \mathrm{e}^{-r \sqrt{i \omega a}}+B \mathrm{e}^{r \sqrt{i \omega a}}
$$

where $A$ and $B$ are coefficients that depend on boundary conditions.

The boundary conditions imply that $B=0$, because the solution must be bounded at infinity. Then

$$
V_{m}=A \mathrm{e}^{-r \sqrt{i \omega a}}
$$

Now we will consider the various transfer functions of the plant defined by different boundary conditions.

\section{A. The Dirichlet (or first-type) Boundary Condition}

Let us consider the case when the input is the temperature of solid surface, and the output is the temperature at the depth $L$.

The boundary conditions of the first kind have the form:

$$
\left.\begin{array}{ll}
X_{m}=V_{m}, & r=0 \\
Y_{m}=V_{m}, & r=L
\end{array}\right\}
$$

The complex gain of the plant $G(i \omega)$ is defined as $Y_{m} / X_{m}$ subject to (10):

$$
G(i \omega)=e^{-L \sqrt{i \omega a}}
$$

The transfer function is 


$$
G(s)=\mathrm{e}^{-\sqrt{s T_{0}}}
$$

where $T_{0}=a L^{2}$. Calculated on the basis of physical data, the value of the time constant is $T_{0}=85.64 \mathrm{~s}$.

Let the input signal changes stepwise from $x\left(0_{-}\right)=288 \mathrm{~K}$ to $x\left(0_{+}\right)=298 \mathrm{~K}$. Then $y\left(0_{-}\right) 288 \mathrm{~K}$. The transient process at the output of the plant is [13]

$$
y(t)=x\left(0_{-}\right)+\left[x\left(0_{+}\right)-x\left(0_{-}\right)\right] \operatorname{erfc}\left[0.5 \sqrt{T_{0} / t}\right] \cdot 1_{0}(t)
$$

where $\operatorname{erfc}(\cdot)=1-\operatorname{erf}(\cdot)$ is the complementary error function,

$$
\operatorname{erfc}(z)=1-(2 / \sqrt{\pi}) \int_{0}^{z} \exp \left(-\xi^{2}\right) d \xi
$$

Let us estimate the value of the time constant by observations of the noisy transition process. The interference signal is a white noise uniformly distributed on the interval $[-1 ; 1] \mathrm{K}$. Normalize the processes so that

$$
\begin{gathered}
x_{N}(t)=\left[x(t)-x\left(0_{-}\right)\right] /\left[x\left(0_{+}\right)-x\left(0_{-}\right)\right] \\
y_{N}(t)=[y(t)-y(0)] /\left[x\left(0_{+}\right)-x\left(0_{-}\right)\right]
\end{gathered}
$$

According to EMM it is necessary to generate one modulating function $\psi(t)=\exp (-t / \theta)$, which at the time of observation would damp to values much smaller than 1 , multiply it on the recorded signals $x(t)$ and $y(t)$ and to calculate the areas under the formed curves:

$$
\begin{gathered}
S_{x}=\int_{0}^{T_{H}} x_{N}(t) \psi(t) d t \approx \int_{0}^{\infty} \exp (-t / \theta) d t=\theta \\
S_{y}=\int_{0}^{T_{H}} y_{N}(t) \psi(t) d t \approx \\
\approx \int_{0}^{\infty} \operatorname{erfc}\left[0.5 \sqrt{T_{0} / t}\right] \exp (-t / \theta) d t
\end{gathered}
$$

Then the estimate of time constant in (13) is

$$
\hat{T}_{0}=\theta \ln \left(S_{y} / \theta\right)^{2}
$$

The estimated value of the time constant is $\hat{T}_{0}=86.41 \mathrm{~s}$. The transient processes at the outputs of the plant and the model obtained through identification are shown in Fig. 2.

\section{B. The Neumann (or second-type) Boundary Condition}

Let the plant input is the heat flow and the output is the surface temperature. Such a situation corresponds to the second-type boundary conditions:

$$
\left.\begin{array}{ll}
X_{m}=-K_{0}\left(d V_{m} / d r\right), & r=0 \\
Y_{m}=V_{m}, \quad r=0 &
\end{array}\right\}
$$

The transfer function is

$$
G(s)=k_{1} / \sqrt{s}
$$

where $k_{1}=1 /\left(K_{0} \sqrt{a}\right)$. Calculated on the basis of physical data, the value of the gain is $k_{1}=2.69 \cdot 10^{-5} \mathrm{~K} \cdot \mathrm{m}^{2} \cdot \mathrm{W}^{-1} \cdot \mathrm{s}^{-1 / 2}$. The transient process is

$$
y(t)=y(0)+2 k_{1} \sqrt{t / \pi} \cdot x_{0} \cdot 1_{0}(t)
$$

where $y(0)=288 \mathrm{~K}$ is the initial temperature on the surface of the ingot, $x_{0}=1 \cdot 10^{4} \mathrm{~W} \cdot \mathrm{m}^{-2}$ is the heat flux density.

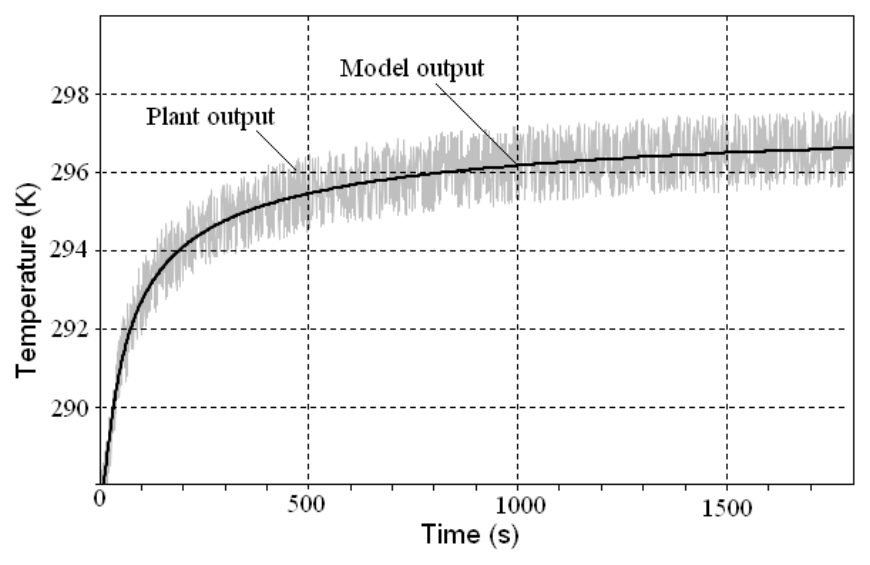

Fig. 2. The transient processes on the plant and model outputs (the Dirichlet boundary conditions)

The estimate of the gain is determined as

$$
\hat{k}_{1}=S_{y} /(\theta \sqrt{\theta})
$$

The calculated value of the time constant according to (23) is $\hat{k}_{1}=2.68 \cdot 10^{-5} \mathrm{~K} \cdot \mathrm{m}^{2} \cdot \mathrm{W}^{-1} \cdot \mathrm{s}^{-1 / 2}$. The transient processes at the outputs of the plant and the model obtained through identification are shown in Fig. 3.

\section{The Robin (or third-type) Boundary Condition}

Let us consider now the case when heat is eliminated from the solid surface. Then the boundary conditions take the form

$$
\left.\begin{array}{l}
X_{m}=-K_{0}\left(d V_{m} / d r\right)+\alpha V_{m}, r=0 \\
Y_{m}=V_{m}, \quad r=0 \quad \text { or } \quad r=l
\end{array}\right\}
$$

where $\alpha=1000 \mathrm{~K} \cdot \mathrm{m}^{2} \cdot \mathrm{W}^{-1}$ is the heat transfer coefficient. The transfer function is

$$
G(s)=k /(1+\sqrt{s T})
$$

where $k=1 / \alpha, T=a\left(K_{0} / \alpha\right)^{2}$ is the unknown gain and time constant. The transient process is

$$
y(t)=k[1-\exp (\mathrm{t} / \mathrm{T}) \operatorname{erfc} \sqrt{t / T}] 1_{0}(t)
$$

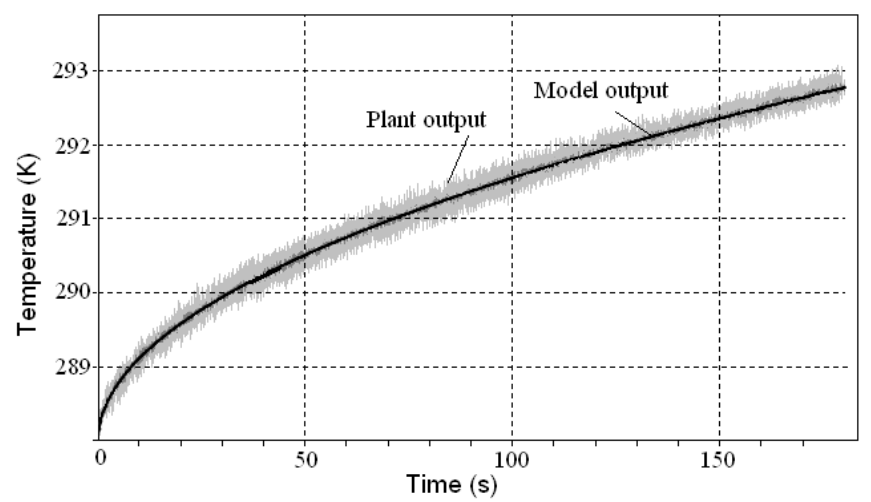

Fig. 3. The transient processes on the plant and model outputs (the Neumann boundary conditions). 
The experimental conditions are the same as in the previous case. Calculated on the basis of physical data, the values of the gain and time constant are $k=1 \cdot 10^{-3} \mathrm{~K} \cdot \mathrm{m}^{2} \cdot \mathrm{W}^{-1}$, $T=439.6 \mathrm{~s}$. In this case there are two unknown parameters, so we need to form two modulating functions $\psi_{1}(t)=\exp \left(-t / \theta_{1}\right)$ and $\psi_{2}(t)=\exp \left(-t / \theta_{2}\right)$ and to calculate two areas $S_{y 1}$ and $S_{y 2}$. Then we can set up the system of equations:

$$
\left\{\begin{array}{l}
\hat{k} /\left(1+\left(\hat{T} / \theta_{1}\right)^{1 / 2}\right)=S_{y 1} / \theta_{1} \\
\hat{k} /\left(1+\left(\hat{T} / \theta_{2}\right)^{1 / 2}\right)=S_{y 2} / \theta_{2}
\end{array}\right.
$$

Solving this system, we get

$$
\begin{gathered}
\hat{k}=\frac{S_{y 1} S_{y 2}\left(\sqrt{\theta_{2}}-\sqrt{\theta_{1}}\right)}{S_{y 1} \theta_{2} \sqrt{\theta_{2}}-S_{y 2} \theta_{1} \sqrt{\theta_{1}}} \\
\hat{T}=\theta_{1} \theta_{2}\left(\frac{S_{y 1} \theta_{2}-S_{y 2} \theta_{1}}{S_{y 1} \theta_{2} \sqrt{\theta_{2}}-S_{y 2} \theta_{1} \sqrt{\theta_{1}}}\right)^{2}
\end{gathered}
$$

The estimates of parameters are $\hat{k}=9.81 \cdot 10^{-4} \mathrm{~K} \cdot \mathrm{m}^{2} \cdot \mathrm{W}^{-1}, \hat{T}=428.2 \mathrm{~s}$. The transient processes at the outputs of the plant and the model obtained through identification are shown in Fig. 4.

Numerical values of estimates and the transient processes shown in Fig. 2-4 demonstrate satisfactory quality of plant identification.

\section{CONCLUSION}

Despite the fact that EMM was originally developed to identify simple plants with lumped parameters, described by rational transfer functions, it turned out to be suitable for identification of objects, described by irrational transfer functions. As follows from expressions (18), (22) and (27), finding the estimates of parameters of the plant cannot be reduced to the solution of a system of linear algebraic equations. In the considered examples we have found analytical solutions for calculating parameter estimates. But for more complex systems it is not always possible to do so. (Examples of such systems can be found, in particular in [8]) In many cases it is necessary to solve the system of nonlinear algebraic equations by numerical methods. However, modern software allows easy enough to solve this problem.

Our future research in this area will be directed, firstly, to solve the problem of identifying objects of a more complex structure; secondly, on the analysis of statistical characteristics of the parameter estimates.

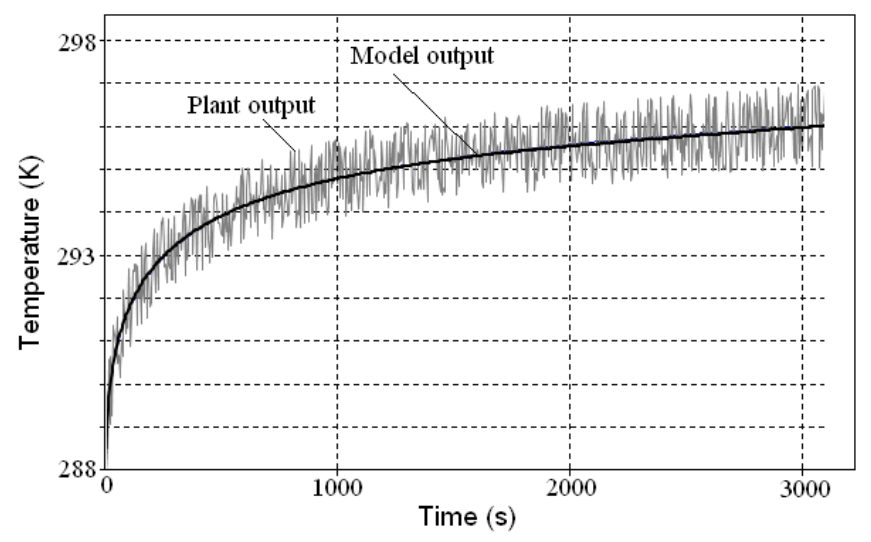

Fig. 4. The transient processes on the plant and model outputs (the Robin boundary conditions)

\section{REFERENCES}

[1] M.S. Tavazoei, "Fractional/distributed-order systems and irrational transfer functions with monotonic step responses," Journal of Vibration and Control, vol. 20, no. 11, pp. 1697-1706, 2014.

[2] D. Sierociuk, A. Dzielinski, G. Sarwas, I. Petras, I. Podlubny, and T. Skovranek, "Modelling heat transfer in heterogeneous media using fractional calculus," Philosophical Transactions of Royal Society A: Mathematical, Physical and Engineering Sciences, A 371: 20120146, April 2013.

[3] S.I. Heizler, "Asymptotic telegrapher's equation $(P 1)$ Approximation for the transport equation," Nuclear Science and Engineering, vol. 166, pp. 17-35, Sept. 2010

[4] N. Kishor, R.P. Saini, and S.P. Singh, "A review on hydropower plant models and control," Renewable and Sustainable Energy Reviews, vol. 11, pp. 776-796, 2007.

[5] M. Soni and S. Kaur, "Modeling of hydraulic turbine for analyzing effect of penstock parameter variation on mechanical power," International Journal of Engineering Research and General Science, vol. 3, no. 3, pp. 604-611, May-June 2015.

[6] T. Hu, B. Zhong, S.L. Dvorak, and J.L. Prince, "Application of recursive convolution to transient simulation of interconnects using a hybrid phase -pole macromodel," IEEE Transactions on Advanced Packaging, vol. 27, no.4, pp. 603-610, Nov. 2004.

[7] J. Tenreiro Machado, and A.M. Lopes, "Dynamical analysis of the global warming," Mathematical Problems in Engineering, vol. 2012, Article ID 971641

[8] R. Curtain, and K. Morris, "Transfer functions of distributed parameter systems: A tutorial," Automatica, vol. 45, pp.1101-1116, 2009.

[9] D. Anisimov, "Identification of linear dynamic plants by the exponential modulation method," Vestnik MEI, no. 2, pp. 74-78, 1994.

[10] M. Shinbrot, "On the analysis of linear and nonlinear systems," Transaction ASME, vol. 79, no. 3, pp. 547-542, 1957.

[11] J.M. Loeb, and G.M. Cahen, "More about process identification," IEEE Transactions on Automatic Control, vol. 10, no. 3, pp. 359-361, 1965.

[12] D. Anisimov, and A. Khripkov, 'Distribution laws of dynamic objects' parameter estimates in the identification by exponential modulation method," Problemy Upravlenija, no. 4, pp. 14-18, 2007.

[13] M. Kolomejceva, and A. Netushil, "The transients in automatic control systems having irrational transfer functions," Avtomatika $i$ Telemehanika, vol. 26, no. 2, pp. 359-364, 1965. 